\title{
Practice and Some Thoughts on a New Peer Reviewer Recommended Mechanism of NSFC: Based on Mutual Trust of the Academic Community
}

\author{
Yafei Dai ${ }^{1}$, Feixue Gao ${ }^{1,}{ }^{\star}$, Cuixia Wang ${ }^{2}$, Yongjun Chen ${ }^{1}$ \\ ${ }^{1}$ Department of Chemical Sciences, National Natural Science Foundation of China, Beijing 100085, P. R. China. \\ ${ }^{2}$ Bureau of Personnel, National Natural Science Foundation of China, Beijing 100085, P. R. China.
}

\section{建立学术共同体互信的基金评审机制的尝试与思考}

戴亚飞 ${ }^{1}$ ，高飞雪 ${ }^{1, *}$ ，王翠霞 ${ }^{2}$, 陈拥军 1

1 国家自然科学基金委员会, 化学科学部, 北京 100085

2 国家自然科学基金委员会，人事局，北京 100085

\begin{abstract}
摘要: 完善评审机制是国家自然科学基金委新时代基金改革的重要任务之一。为切实落实该项改革任务, 建立符合新时 代科学基金资助导向的科学、公正、高效的评审机制, 基金委化学部努力探索新的学科基金管理模式, 尝试了在通信评 审阶段由科学家与学科管理人员共同参与项目的分组、指派, 共同完成同行评议专家遴选工作的新模式。本文分析了科 学家参与同行评议专家遴选工作的利弊, 探讨了如何更好的发挥科学家在同行评议专家遴选工作中的作用, 建立学术共 同体互信的基金评审机制。
\end{abstract}

关键词：同行评议；专家遴选；学术共同体；基金评审机制

建立科学、公正和高效的评审机制一直以来 都是国家自然科学基金委员会(以下简称基金委) 的宗旨, 新时代科学基金改革再次明确提出要进 一步完善评审机制 ${ }^{1}$ 。长期以来，基金委项目的规 划、立项、评审、中期检查和结题审查等环节主要 采用函评和会评的同行评议制 ${ }^{2-4}$ 。而在同行评议 过程中, 同行评议专家的遴选起着至关重要的作 用, 只有遴选出高水平的同行评议专家, 才有可能 对项目作出科学、客观和公正的评价。为认真落实 基金委新时代基金改革试点工作，进一步提升同 行评议的科学性、公正性和公平性, 营造风清气正 的学术环境, 建设科学家与基金管理人员互信的 良好学术生态, 建立符合新时代科学基金资助导 向的评审机制, 基金委化学部与人事局共同努力 探索了学科基金评审与管理新模式, 从通讯评审 阶段的同行评议专家遴选工作入手, 对学科基金
项目的评审与管理工作进行了试点改革。

1 完善基金评审机制的背景、目的和意义

通讯评审阶段的同行评议专家遴选对能否科 学、客观和公正的遴选出高质量的项目起着至关重 要的作用, 专家的个人认知、学术水平以及对项目 内容的熟悉程度均会影响到对项目的学术判断。如 何为每一个项目准确地遴选出合适的同行评议专 家是一个值得探讨的问题。现阶段基金委的同行评 议专家遴选的常规程序是由学科项目主任将研究 内容相近的项目进行分组, 再按照研究领域相近的 匹配原则, 结合相关回避原则, 通过计算机辅助指 派系统将每组项目指派给小同行评审专家进行通 讯评审。一直以来, 尽管学科管理人员作出各种不 懈努力追求项目评审的科学性、公平性和公正性, 但总不可避免地出现少数同行评议专家并没能给

Received: March 13, 2020; Revised: March 16, 2020; Accepted: March 16, 2020; Published online: March 16, 2020.

*Corresponding author. Email: gaofx@nsfc.gov.cn; Tel.: +86-10-62327035.

(C) Editorial office of Acta Physico-Chimica Sinica 
出科学、客观和公正的评价。究其原因可能有以下 几方面: 1) 基金委实行轮岗制, 在同一岗位工作 六年及以上必须从目前的工作岗位轮换到另一个 岗位。通常学科项目主任从一个学科轮岗到另一 学科, 总需要一个熟悉和适应的过程, 很难迅速掌 握新学科的前沿领域和学科内容及布局, 该学科 的专家队伍情况, 以及相关专家的具体研究内容 等, 这可能导致了所遴选的通讯评审专家与项目 的研究内容匹配的不够精准, 专家对项目的研究 内容不够熟悉, 对项目的理解不够透彻, 从而没有 能力给出科学的评价; 2) 目前基金委受理的项目 数量逐年攀升, 2019年全委接收到的项目申请数 目已经突破 25 万, 而基金委正式工作人员加流动 项目主任只有不到 500 人, 在有限的时间内要完成 巨大数量的项目指派工作, 人力资源严重短缺。如 此高强度的工作也导致学科工作人员难以保证有 足够的时间对每一个项目通讯评审专家的遴选进 行充分的勘酌, 不可避免的会出现个别项目的通 讯专家遴选存在偏差；3）同行评议专家在评审过 程中可能受到各种非科学因素的干扰而没有给出 科学、客观和公正的评价等。

基于以上原因, 化学科学部尝试了在通讯评 审阶段邀请科学家参与项目的通讯评审专家遴选 工作的基金项目管理新模式。邀请相关研究领域 的科学家参与通讯评审专家的遴选工作, 一方面 可以充分利用委外智力资源, 发挥科学家的专业 优势, 提升基金管理绩效; 另一方面也解决基金委 学科人力资源严重不足的问题。同时, 希望通过本 次试点工作, 让科学家深入了解基金委的工作流 程, 切身体会基金委追求科学、公平和公正的评审 理念, 与基金委工作人员一起, 共同营造风清气正 的学术环境。

\section{2 科学家参与通讯评审专家遴选工作的试 点实施}

为确保科学家参与通讯评审专家遴选试点工 作的顺利进行, 基金委化学部会同人事局和信息 中心等部门进行了多次协商和研讨, 综合考虑基 金委相关人事制度、回避制度和学科特征以及试 点工作的实施时间等, 最终确定了参与本次试点 工作的学科和科学家人选。在此基础上, 又制定了 既能让科学家与学科管理人员互相信任又能互相 制约的具体实施方案。

本次工作选取了化学科学部“化学理论与机 制”学科中“理论与计算化学(B0301)”领域的面上 项目作为试点学科。选择该领域作为试点的原因
主要是考虑到该领域中研究方向相对集中, 便于 初次试行与摸索经验。实际执行过程中，根据该领 域项目类型的特征, 共邀请了理论化学领域的三 位科学家参加了试点工作, 这三位科学家长期以 来在学术界有着良好的口碑和声誉, 学术造诣好、 学术视野宽、学风端正和为人正直。另外, 近五年 他们主持过重点、重大、重点国际合作、创新群体 等类型项目, 确保了他们对该领域研究方向的项 目定位、组成分布以及该领域专家分布情况都有 充分的了解。

根据实施方案, 本次试点工作以会议的形式 进行。三位科学家在指定时间到会, 到会之前他们 对会议的内容并不知晓。到会后人事局、科学部和 科学家共同签订了保密承诺书, 会场也做了各项 保密措施。会议期间学科管理人员全程陪同, 随时 解决科学家指派过程中遇到的技术问题, 但学科 管理人员不得干预科学家的指派工作, 科学家之 间也不得交流, 所有指派工作需独立完成。按实施 方案的具体要求, 每位科学家对各自负责的研究 领域项目进行分组, 每一组项目遴选6位通讯评审 专家, 再由学科管理人员从中挑选 3 位, 完成最终 的项目指派。整场工作进行的非常顺利, 全部指派 工作在一天半时间内圆满完成。

\section{3 对科学家参与通讯评审专家遴选试点工 作的分析与建议}

试点工作结束之后，学科管理人员和科学家 对本次工作进行了研讨和总结, 一致认为科学家 参与通讯评审专家的遴选工作在以下几个方面发 挥了重要作用:

1) 科学家的参与在某种程度上减少了学科 工作人员的工作量, 减轻了工作压力;

2) 科学家对同领域评审专家的研究方向了 解更深, 有助于进一步提升同行评审的科学性;

3) 试点工作为科学家和学科管理人员提供 了深入交流的平台, 一方面为科学家提供了了解 学科管理工作的机会; 另一方面科学家从专业的 视角对学科管理工作也提出了建议, 有利于进一 步完善基金评审机制;

4) 试点工作增加了科学家和学科管理人员 之间的互助互信, 进一步营造了风清气正的学术 环境。

目前, 将此次试点工作进行大面积推广, 还存 在一定的局限性。主要是因为面上类项目涉及研 究领域非常宽泛。与学科管理人员相比, 科学家更 多专注于与自己工作相近的领域, 对其他领域和 
相关专家的了解和熟悉程度不够。从本次试点情 况看, 三位科学家花了一天半的时间才完成 164 个 项目的分组和通讯评审专家的遴选工作。如果由 学科项目主任操作, 一人一天半即可完成。另外, 即使有计算机辅助指派系统协助, 由于熟悉程度 不够，三位科学家仍然花费了很长时间从计算机 匹配出来的专家中进行二次挑选，并且明确表示 对部分项目的专家遴选不是十分有把握。此外, 科 学家工作繁忙且时间宝贵, 很难保证连续几天时 间参与到基金委项目管理工作中来，因此全程参 与到大量面上类项目通讯评审专家遴选工作存在 现实的困难。

综上所述，科学家与基金委管理人员共同参 与通讯评审专家的遴选工作, 是一种值得肯定和 有效的通讯评审专家遴选模式。这种指派方式可 以试用于申请数量不多、研究领域分布相对集中 的项目类型或者具有交叉学科性质的项目评审中
推广, 比如重大研究计划、重大仪器研制项目、联 合基金项目和学科领域相对集中的面上项目等。

建立科学、公正和高效的评审机制需要长期 探索和不解努力。基金人只有勇于担当新时代科 学基金改革工作的任务, 通过不断尝试和改进, 逐 步完善基金项目评审工作机制。

\section{References}

(1) Li, J. H. Bull. Natl. Nat. Sci. Found. China 2018, 32 (4), 345. [李静 海. 中国科学基金, 2018, 32 (4), 345.]

(2) Rothenberg, M. Technol. Innov. 2010, 12, 189.

(3) Yao, Y. P. Bull. Natl. Nat. Sci. Found. China 2017, 31 (4), 346. [姚玉 鹏. 中国科学基金, 2017, 31 (4), 346.]

(4) Jiang, H. J.; Hao, Y. N.; Xu, Y. Y.; Sun, R. J. Bull. Natl. Nat. Sci. Found. China 2019, 33 (2), 149. [江虎军，郝艳妮，徐岩英，孙瑞娟. 中国科学基金, 2019, 33 (2), 149.] 